\title{
Protecting the Health Service and Managing the COVID-19 Pandemic Surge: The Unintended Impact of Service Reconfiguration on the Specialist Rehabilitation Service at a Regional Teaching Hospital
}

\section{Cosgrove $T^{\mathbf{1}}$ and Salawu $A^{1,2 *}$ \\ ${ }^{1}$ Department of Rehabilitation Medicine, Hull University Teaching Hospitals NHS Trust (HUTH), UK ${ }^{2}$ Hull York Medical School (HYMS), UK \\ *Correspondling author: Salawu A, Department of Rehabilitation Medicine, Hull University Teaching Hospitals NHS Trust (HYMS), UK}

Received: January 14, 2021; Accepted: February 27, 2021; Published: March 06, 2021

\begin{abstract}
As part of the UK National Health Service (NHS) preparations to manage the impact of the COVID-19 pandemic on hospital services, clinical guidelines that emphasized the expansion of the acute care capacity in managing the anticipated surge in COVID-19 cases were implemented. Clinical wards were reconfigured and routine face-to-face outpatient clinics were suspended. Some of the changes include workforce and facilities reorganization. One of the changes implemented at the Hull University Teaching Hospitals NHS Trust (HUTHT), was the relocation of the Complex Rehabilitation Ward from its 15bed base in the Queen's Centre for Oncology to a repurposed 12-bed surgical ward within the main Castle Hill Hospital $(\mathrm{CHH})$ building in March 2020.
\end{abstract}

Methods: A comparative review of the admissions and outcome measures data (admission diagnosis; referral source; PCAT: Patient Categorization Tool; LOS: Length of Stay; Bed occupancy and discharge destinations) over a 4-month period (March-June 2020) was undertaken and compared to retrospective data from a corresponding 4-month (March-June 2019) period in the previous year to determine the impact of the ward relocation on the delivery of specialist rehabilitation to patients with complex needs during the pandemic episode.

Results: A reduction in total number of admitted patients $(n=28$ in 2019; $\mathrm{n}=18$ in 2020) with reduced bed occupancy from $99 \%$ in 2019 to $72 \%$ in 2020 despite a reduction in bed base was noted following the ward relocation. A shorter length of stay with a mean of 29 days was noted following relocation of the ward while an increase in patient complexity as demonstrated by the PCAT scores was observed. The proportion of patients achieving a home discharge destination as opposed to other residential care facilities increased, accounting for $89 \%$ among discharged patients.

Conclusion: This review demonstrated some of the impact of the measures implemented to combat the 1st wave of the coronavirus pandemic, specifically the relocation of the specialist inpatient rehabilitation ward in a tertiary hospital setting. Though a higher proportion of the admitted patients had increased complexity, a shorter length of stay with a significant proportion of the patients achieving a home discharge destination were observed following ward-relocation in 2020 compared to a similar quarter in 2019. The findings also reflected a reduction in bed occupancy despite reduced bed base capacity. Though there is a noted reduction in duration of stay in hospital and a greater proportion the patients achieved a home discharge destination, this was achieved with a compromise on the rehabilitation process due to the constraints of the new ward environment. Significant impact in the quality of the therapy programmes delivered was observed. The longer-term impact of this will need to be monitored. This review highlights the need for consideration of specialist rehabilitation as part of the acute response planning process in pandemic and mass casualty events.

Keywords: COVID-19; Complex Rehabilitation; Hospital service reconfiguration; Patient discharge; Acute Rehabilitation
Phys Med Rehabil Int - Volume 8 Issue 1 - 2021 ISSN : 2471-0377 | www.austinpublishing group.com Salawu et al. (C) All rights are reserved
Citation: Cosgrove T and Salawu A. Protecting the Health Service and Managing the COVID-19 Pandemic Surge: The Unintended Impact of Service Reconfiguration on the Specialist Rehabilitation Service at a Regional Teaching Hospital. Phys Med Rehabil Int. 2021; 8(1): 1175. 


\section{Introduction}

Cases of a novel respiratory infection caused by a newly identified virus, SARS-COV-2 belonging to the coronavirus family were first reported in the Wuhan district of China and notified to the WHO on the $31^{\text {st }}$ of December 2019 [1]. The disease caused by this virus was labelled COVID-19 by the WHO on 11 February 2020. A majority (81\%) of those infected with the virus have a mild/asymptomatic infection whilst $14 \%$ develop moderate to severe illness that requires hospitalization. Roughly, 5\% go on to develop critical illness requiring ventilatory support and management in the intensive care unit [2]. Certain health conditions increase the susceptibility to developing severe/critical illness following infection with the virus. These conditions include obesity, insulin resistance and diabetes mellitus, cancer, chronic obstructive pulmonary disease, chronic kidney disease, sickle cell disease, heart failure and immunocompromised state [3].

The first reported UK cases were identified in York within our region on the $31^{\text {st }}$ January 2020 in 2 members of the same family who were visiting the UK from China [4]. Subsequently UK infection and mortality figures have increased exponentially to 284,900 confirmed cases and 44,198 reported deaths as of the $4^{\text {th }}$ of July 2020 [4]. As the number of cases increase, the government introduced a range of measures to limit the spread of the infection and to ensure that health care service capacity is not overwhelmed culminating in a lockdown on the $23^{\text {rd }}$ of March 2020 [4].

National guidelines were developed with the aim to create extra capacity for the anticipated surge in admissions at the peak of the pandemic. The guidelines required various bodies such as the UK National Health Service (NHS), Hospital Trusts, Clinical Commissioning Groups (CCG), and Social Services to work together in achieving this. Elective clinical activities including outpatient clinics and procedures were suspended and workforce reorganization with staff redeployment especially among clinical staff undertaken to ensure frontline services will not be overwhelmed. Additional measures include the implementation of virtual clinics with the use of telephone and video consultation facilities. Facilities reconfiguration included closure and relocation of some ward services based on the emerging evidence that some conditions increases the vulnerability to contracting and developing severe form of the COVID-19 disease.

Within HUTHT, implementation of this guidance influenced the Specialist Rehabilitation service that provides multidisciplinary rehabilitation to patients with complex clinical conditions and disabilities. The Complex Specialist Rehabilitation ward (C29) is a 15-bedded unit located within the Queens centre for Oncology and Hematology at Castle Hill Hospital (CHH). The Queens Centre for Oncology and Hematology is a self-contained detached building comprising of 5 wards, radiotherapy suites and outpatient facilities in addition to office accommodation for the oncology service. The rehabilitation ward C29 is served by a suitably equipped therapy gym located adjacent to it. This ward was vacated for the relocation of the Oncology Day Assessment Service to ward 29 while the Complex Specialist Rehabilitation ward was relocated to the Elective Orthopedic Surgical ward (C9a) within the same hospital. The aim was to ensure that patients on admission with oncological diagnosis are placed in a bubble within the Queens Centre mainly comprising of cancer-related services. Though it can be argued that the patient group served by the complex rehabilitation service fall within those vulnerable to the COVID-19 disease, a relocation to main hospital building on the Castle Hill Hospital site was non the less implemented.

A change in the rehab ward location brought drastic alterations to accommodation, with requirement on the Complex Rehabilitation Service to adapt patient rooming to satisfy the constraints of the environment. Bed numbers dropped from 15 to 12 , and a 4-bedded bay converted into a makeshift ward gym. To compare the accommodation facilities between the wards, ward C29 offered 9 single occupancy cubicles, with 3 dual occupancy bays. All rooms were equipped with ensuite facilities \& showers. In contrast, C9a provides 4 smaller sized single occupancy cubicles and 2 four-person bays. Within C9a there are two patient toilets in common areas, one shower room, and two cubicles equipped with ensuite toilets without showers.

This narrative aims to explore and to measure the impact of relocation of the Specialist Rehabilitation service through a review of routine outcome measures collected and submitted to the United Kingdom Rehabilitation Outcome Collaborative (UKROC). In addition, this review enabled a comparison of the length of stay and discharge destination for the periods covered. We aim to apply the findings to inform future deliberations on service reconfiguration and relocation decisions on provision of this essential service.

\section{UKROC}

The UK Rehabilitation Outcomes Collaborative (UKROC) is a national database developed to collate inpatient case episodes for patients admitted to UK Rehabilitation services [5]. The HUTHT Complex Rehabilitation Service is a member of the collaborative and submit basic monthly data on admissions and outcome measures (PCAT, RCS and FIM/FAM) to the UKROC.

\section{PCAT}

Patient Categorisation Tool (PCAT) is a clinical checklist document completed on admission to a rehabilitation service, detailing specific scoring criteria relating to the overall clinical impression of patient needs (ranked in order of reducing severity as A, B or C). This helps inform the level of rehabilitation service designated as levels1, 2 or 3 in order of decreasing specialization required based on the British Society of Rehabilitation Medicine (BSRM) service standards [6]. PCAT was originally designed to provide a descriptive measure of patient needs, it has since been refined for use as an ordinal tool in associating rehabilitation needs to patient pathology [7].

\section{Materials and Methods}

Admissions and outcomes data were collected in the first four months of the HUTHT Complex Rehabilitation Service relocation to C9a (March-June of 2020), a period coinciding with the $1^{\text {st }}$ peak of coronavirus infections and hospital admissions for COVID-19 in the UK [4].

To contextualize this information, the C9a data was compared with retrospective data from the corresponding period in 2019 from C29 (previous ward). Direct comparison of patient discharge data allowed the team to examine length of stay and patient complexity in two equivalent periods. 
Table 1: Demographics for patients admitted in the March-June 2019 \& MarchJune 2020 quarters.

\begin{tabular}{|c|c|c|}
\hline & March-June 2019 & March-June 2020 \\
\hline Male & $21[72 \%]$ & $12[66 \%]$ \\
\hline Female & $8[28 \%]$ & $6[33 \%]$ \\
\hline
\end{tabular}

Table 2: Monthly [mean average] representative percentage occupancy in both study periods.

\begin{tabular}{|c|c|c|}
\hline $\begin{array}{c}\text { Month of } \\
\text { Study }\end{array}$ & $\begin{array}{c}\text { Month average percentage } \\
\text { occupancy [\%] }\end{array}$ & $\begin{array}{c}\text { Month average percentage } \\
\text { occupancy [\%] }\end{array}$ \\
\hline March & 100 & 73 \\
\hline April & 98 & 82 \\
\hline May & 100 & 62 \\
\hline June & 100 & 69 \\
\hline
\end{tabular}

\section{Results}

\section{Admissions data}

Data was obtained from the records of a total of 47 patients ( $n=29$ [21 males: 8 females] in the March-June 2019 cohort; $\mathrm{n}=18$ patients [12 Males; 6 Females] admitted from March-June 2020) (Table 1).

\section{Capacity and occupancy (Table 2 and Figure 1)}

Referring specialties (Figure 2)

Admission diagnosis and indications (Figure 3)

Patient complexity data (Figure 4)

Length of stay (Figure 5)

Discharge destinations (Figure 6 and 7)

\section{Discussion}

According to the BSRM [8]; Specialist Rehabilitation is defined as the total active care of patients with a disabling condition, and their families, by a multi-professional team who have undergone recognized specialist training in Rehabilitation, led or supported by a consultant trained and accredited in rehabilitation medicine. Specialist Rehabilitation Services provide specialist medical and therapeutic care of challenging patient presentations to support recovery using a multi-disciplinary bio-psycho-social model8. Increasingly, the value of Rehabilitation is being recognized as an integral component of the patient journey in facilitating safe and effective discharge for the deconditioned and significantly deteriorated patient [9]. Figure 1 and Figure 2 give some indication as to the variety of patient referrals encountered by the Service at HUTHT, demonstrating a greater variety of patient presentations and referring specialties in 2020. This increase in variety may be explained by the service-wide reconfiguration of wards within HUTHT and the shifting attitudes to patient management caused by the COVID-19 pandemic.

Specialist Rehabilitation beds are a finite resource under increasing demand in UK hospitals [10]. Locally, the HUTHT Specialist Rehabilitation Service with 15 bed-spaces, serves a population of 1.5 million residents. This represents a regional shortfall of 52-82 beds based on the recommendations of the British Society of Rehabilitation Medicine [11]. The subsequent relocation to ward C9a, with 12 bed spaces, effectively increased the shortfall to $55-85$ beds. To mitigate against this, a multidisciplinary Acute Rehabilitation Team (ART) was created in 2018 to provide peripatetic input to patients on acute

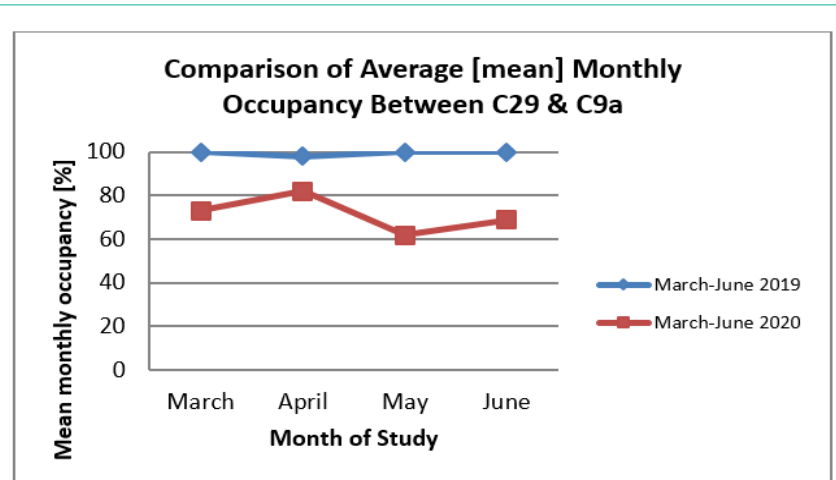

Figure 1: Mean monthly occupancy (data from Table 2) represented for comparison.

wards awaiting transfer to the specialist Rehabilitation ward. The use of a waiting list in subacute specialty services is well-established [12], with prioritization and triage held as key cornerstones of patient-flow management $[13,14]$. Waiting lists may potentially adversely affect patient outcomes if access is delayed [15]. The HUTHT ART further benefits patients through provision of additional rehabilitation input whilst accepted to the waiting list, augmenting Therapies delivery on these wards, and many patients are optimized to an effective level promoting safe discharge from hospital without the need for transfer. It is important to highlight that admissions data does not include the gross number of patients referred.

Admission Figures in Table 1 indicate fewer patients were admitted in 2020 despite an increase in referring specialties (Figure 2). Referrals were received from 10 specialties in comparison to 8 in the previous year. When admission data were compared with bed occupancy (Figure 1 and Table 2), there is a reduction in number of patients referred for inpatient rehabilitation when compared with 2019 data.

HUTHT service reconfiguration not only impacted on the Complex Rehabilitation Service. Trust guidance fostered changes in patient flow to ICU where patients with active COVID-19 infection, once stable, were stepped-down infectious Diseases (ID) ward. Early indications suggested Rehabilitation departments would be overwhelmed due to predicted patient volume of post-COVID patients [16], however this has not been demonstrated in this review. In fact, as is seen in Figure 2. There were 0 referrals from ICU with only one patient treated in the 2020 cohort for post-COVID pneumonitis (Figure 3) transferred from the infectious disease ward.

The patient categorization tool (Figure 4) demonstrated a greater proportion of the patients admitted to ward C9a during the period March-June 2020 had higher complexity compared to those admitted to ward C29 in the previous year. The referrals data noted in Figure 3 also reflect this. This trend likely reflects the prevailing situation within the hospital as the number admitted patients with COVID-19 started to rise. Many patients from the surgical and medical wards were discharged from hospital and those who were not medically fit for discharge and have rehabilitation potential were considered for transfer to the specialist rehabilitation ward for ongoing medical care and rehabilitation. This movement of patients from the acute care wards helped to create an enhanced bed capacity within the hospital 


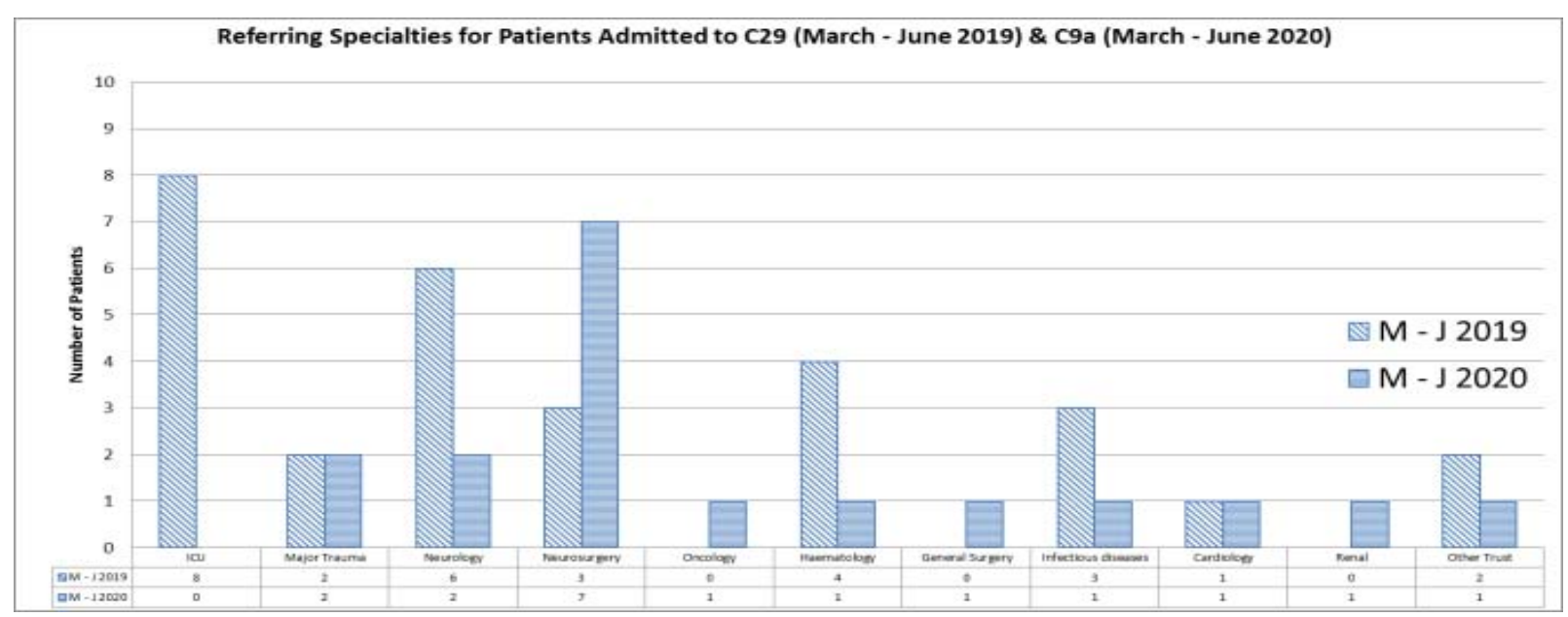

Figure 2: Sources of referral of admitted patients to wards C29 [2019] \& C9a [2020].

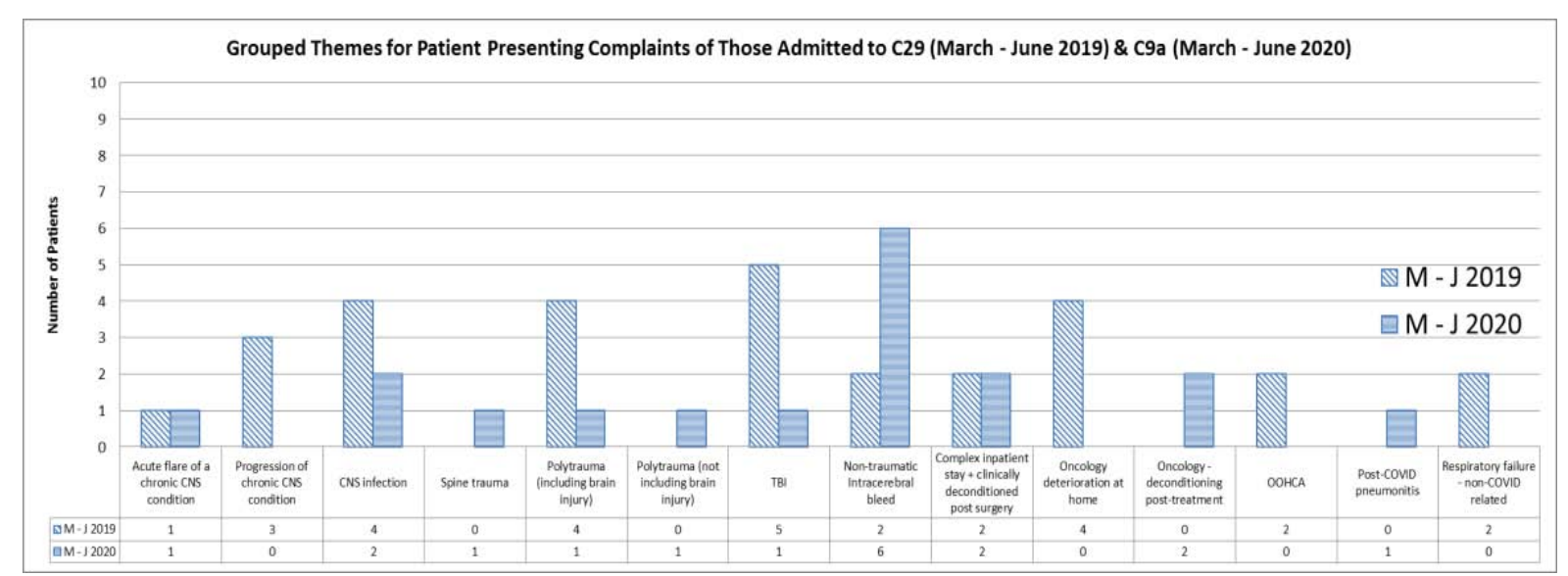

Figure 3: Indications for admission to both C29 in March-June 2019 \& C9 in March-June 2020.

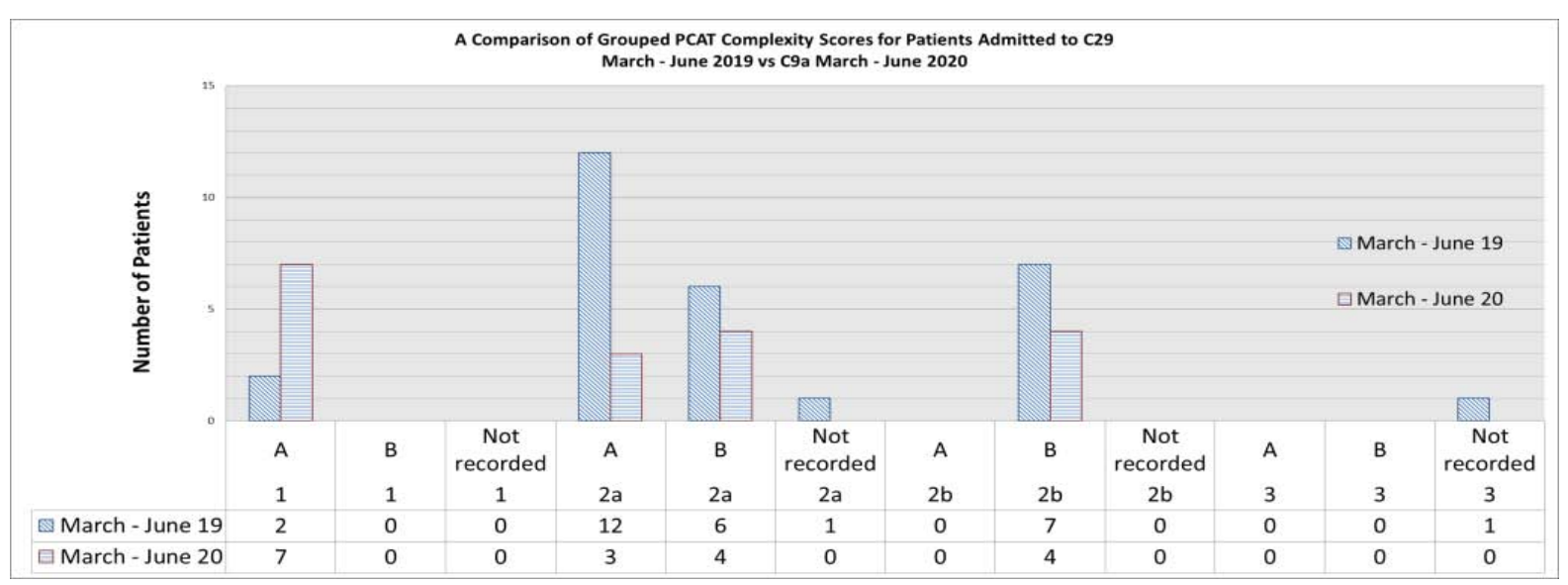

Figure 4: Patient Categorisation Tool (PCAT) data as a measure of patient complexity comparison between 2019 \& 2020.

to deal with anticipated rise in patients with COVID-19. Despite this observed trend with increasing medical complexity of admitted patients to the rehabilitation ward, there is a reduction in length of stay with a mean of 29 days on the rehabilitation ward when compared to data from 2019 with a mean length of stay of 70 days (Figure 5). In addition, a greater proportion of these patients achieved a home discharge destination (Figure 6 and 7) from C9a (89\%) compared to $55 \%$ achieving a discharge back home from C29 in the corresponding 


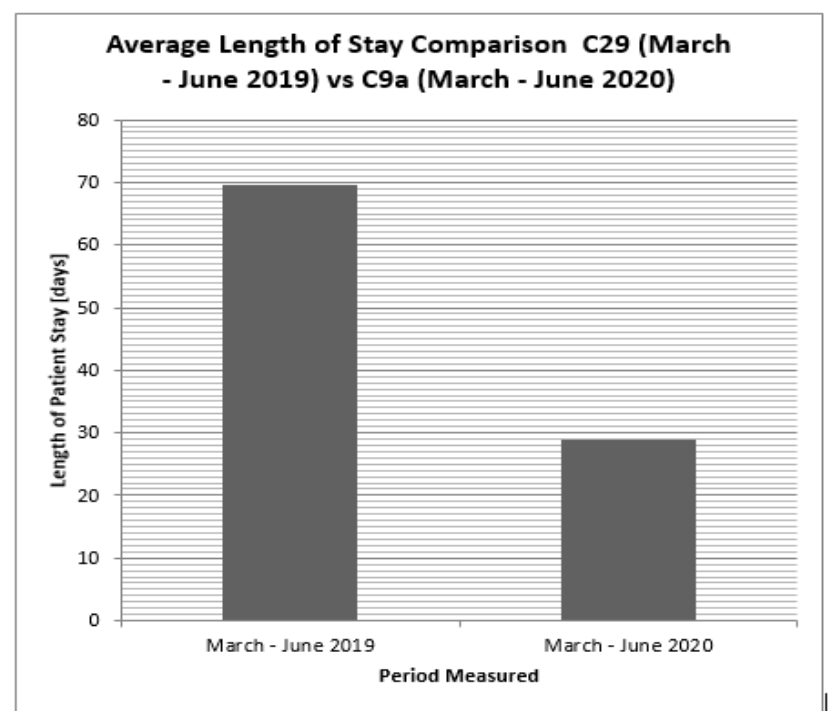

Figure 5: Length in days for patients admitted between March-June 2019 [C29] \& March-June 2020 [C9a].

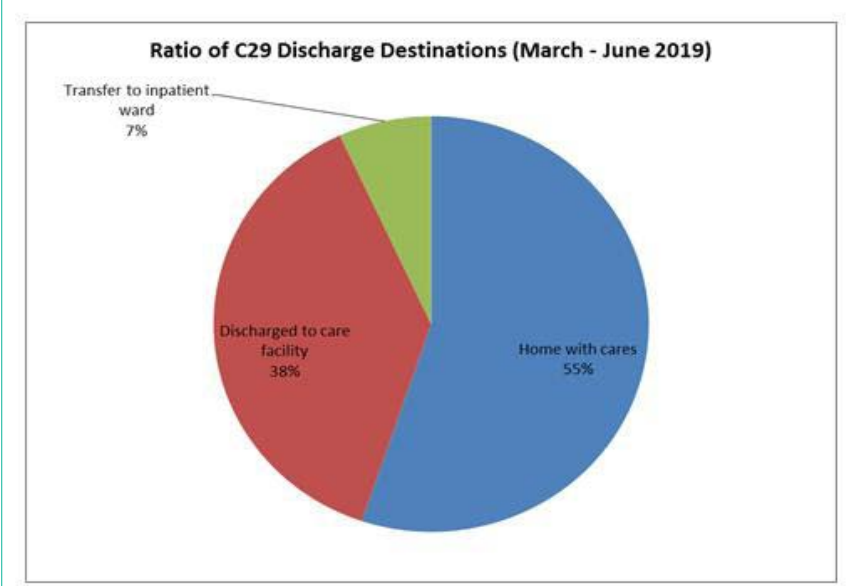

Figure 6: Patient discharge destinations represented as a percentage for comparison in patients admitted to C29.

period of 2019.

A possible contributor to the reduction in the length of stay is the increased use of the discharge to assess pathway model [17]. Discharge to Assess (D2A) model is a concept whereby patients are transferred from acute hospital at the point where they no longer require acute hospital care. The model acknowledges that though a patient might be medically stable, they however might still require care services either for the short or longer term. This care requirement is often a cause of delayed discharges from hospital. The discharge to assess model identifies 4 clear pathways for discharging patients from hospitals. Thereafter any further health or social care assessments are delivered outside of the acute hospital environment. Pathway 0 acknowledges that $50 \%$ of patients will be discharged from hospital without requiring any further input from health and care services. About $45 \%$ of patients discharged with require some form of support from health/care services either at home (Pathway 1). The rest (4\% and $1 \%$ respectively) will require support in community based subacute bed with rehab and re-enablement (Pathway 2) or in a care home sub-acute bed with recovery and complex assessment (Pathway 3).

Another factor that could have contributed to increased home discharges, is the realization that the rate of Coronavirus infection in the community, especially residential care facility was rising at this period thereby making many patients elect to be discharged back to their own home. Additionally, with the lockdown in place and many people working from home, there seems to be support from family members readily available for the patients within their own home.

Whilst the data is reflective of a system-wide shift in patient complexity and outcomes, it is pertinent to know and acknowledge the impact of the change in the ward environment of the quality of rehabilitation input provided to the patients.

COVID-status was used as an indicator of bed placement in transfer to C9a. Patients with negative swabs for COVID-19 were transferred to open-bays, whilst those with positive PCR results were transferred to the side rooms. Despite rigorous application of quarantine principles, five inpatients swabbed positive for SARSCOV-2 (nosocomial infection) during the course of their admission, four of whom had been nursed in 4-bedded bay, therefore forcing bed-closures further reducing capacity. Patients nursed isolation cubicles were unable to access the wider rehabilitation facilities during the period of quarantine.

Under infection-control guidance COVID-19-postive patients must be isolated, with strict PPE measures in place, to ensure nosocomial spread is limited (REF). However, strict isolation should be avoided in a Rehabilitation environment due to Therapies requirements $[18,19]$. Direct comparison of ward configurations highlights a relative paucity of isolation spaces in C9a, comprising of two 4-bedded bays \& four cubicles, versus C29 (9 single occupancy cubicles, with 3 dual-occupancy rooms). The lack of individualized toileting \& showering facilities further compounds this issue, increasing risk of nosocomial infection [20] \& potentially presenting barriers to self-actualised care-needs [21,22].

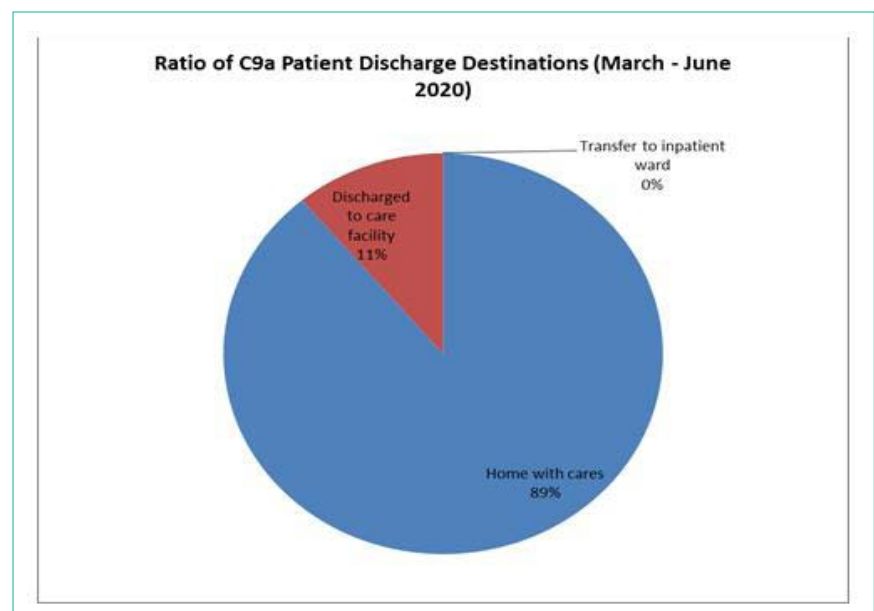

Figure 7: Patient discharge destinations represented as a percentage for comparison in patients admitted to C29. 
Relocation of the service unfortunately impacted the delivery of Physical \& Cognitive Therapies, most notably through the loss of the dedicated gymnasium and assessment area. Within C29, Physiotherapists worked to deliver bespoke regimes using a spacious gym equipped with cutting-edge physical therapy equipment. Since transition to $\mathrm{C} 9 \mathrm{a}$ this gym space has been radically reduced in size, with basic equipment, confined to a previously empty four-bedded bay on an adjacent ward, a downgrade that is likely to impair delivery $[23,24]$.

Despite these barriers to effective rehabilitation, there is an observed reduction in patient length of stay from C29 vs. C9a (Figure 5) there is some weight to the argument of reduced intensity of rehabilitation in the March-June 2020 cohort due to reduced access to quality equipment leading to earlier discharge, however on its own this does not correlate well when discharge destinations are compared as a greater proportion $(89 \%$ vs. $55 \%)$ of patients returned to their home with cares. Instead, when this is framed in the context of the unfolding pandemic, this may be explained as a means to decant patients to their home environment as a means to shield patients from the risk of contracting COVID-19. Pressures exerted by the pandemic was also reflected in the falling proportions of referrals to care facilities ( $11 \%$ vs. $38 \%)$ and those requiring transfer to inpatient wards $(0 \% v s .7 \%)$ however these shifts may be explained by patients wishing to avoid potential exposure to COVID-19.

\section{Conclusion}

This service review has highlighted some of the impact that measures implemented within the health service to protect acute services had on specialist inpatient rehabilitation facility at a large teaching hospital in the UK. It highlights the need for rehabilitation to be considered as a key component in the planning of the health care response to the pandemic and similar future episodes. Interestingly, a higher proportion of home destination for patient discharge was achieved from the constrained environment of the new ward C9a compared to the previous specialist ward location C29. This however may be a reflection of the prevailing circumstance at the peak of the pandemic in UK where infection and mortality rates from COVID-19 were higher in community placement destinations. Patients and their relatives therefore opted for a home destination discharge. The lockdown restriction also meant most households have additional supportive relatives at hand.

\section{Recommendations}

This discussion has demonstrated that in the event of similar event in future, the needs of patients undergoing Rehabilitation should be a key consideration in the plans to relocate such services. It is recommended to provide adequate patient facilities and amenities such as toilets \& showers, ideally in ensuite rooms to reduce the risk of contamination.

Such plans should include the development of individualized treatment plans for post-pandemic patients, with optimization for intense 'short-stint' rehabilitation to maximize potential prior to discharge. In addition, adequate space for gym equipment and assessment spaces should be available with access to common patient area such as a dedicated day room, for patients to engage with supplementary facilitated activities.

\section{References}

1. Coronavirus Disease (COVID-19): Events as they happen. The World Health Organisation. 2020

2. Wu Z, McGoogan J. Characteristics of and Important Lessons from the Coronavirus Disease 2019 (COVID-19) Outbreak in China. Journal of the American Medical Association. 2020; 323: 1239-1242.

3. Coronavirus Disease 2019 (COVID-19). Center for Disease Control and Prevention. 2020.

4. Covid-19 pandemic: Tracking the global coronavirus outbreak [Internet]. BBC News. 2020.

5. Turner-Stokes L, Poppleton R, Williams H, Schoewenaars K, Badwan D. Using the UKROC dataset to make the case for resources to improve costefficiency in neurological rehabilitation. Disability and Rehabilitation. 2012; 34: 1900-1906.

6. Siegert R, Medvedev O, Turner-Stokes L. Dimensionality and scaling properties of the Patient Categorisation Tool in patients with complex rehabilitation needs following acquired brain injury. Journal of Rehabilitation Medicine. 2018; 41: 435-443.

7. Turner-Stokes L, Krägeloh C, Siegert R. The patient categorization tool: psychometric evaluation of a tool to measure complexity of needs for rehabilitation in a large multicentre dataset from the United Kingdom. Disability and Rehabilitation. 2018; 41: 1101-1109.

8. BSRM Standards for Rehabilitation Services Mapped on to the National Service Framework for Long Term Conditions. British Society of Rehabilitation Medicine. 2020.

9. Ross D, Heward K, Salawu Y, Chamberlain M, Bhakta B. Upfront and enabling: Delivering specialist multidisciplinary neurological rehabilitation. International Journal of Therapy and Rehabilitation. 2009; 16: 107-113.

10. Duarte A, Bojke C, Cayton C, Salawu A, Case A, Bojke L, et al. Impact of specialist rehabilitation services on hospital length of stay and associated costs. The European Journal of Health Economics. 2017; 19: 1027-1034

11. Medical Rehabilitation in 2011 and Beyond. British Society of Rehabilitation Medicine \& the Royal College of Physicians. 2020.

12. Harding K, Robertson N, Snowdon D, Watts J, Karimi L, O'Reilly M, et al. Are wait lists inevitable in subacute ambulatory and community health services? A qualitative analysis. Australian Health Review. 2018; 42: 93.

13. Harding K, Taylo N, Leggat S. Do triage systems in healthcare improve patient flow? A systematic review of the literature. Australian Health Review. 2011; 35: 371.

14. Passalent C, Landry M, Cott C. Exploring Wait List Prioritization and Management Strategies for Publicly Funded Ambulatory Rehabilitation Services in Ontario, Canada: Further Evidence of Barriers to Access for People with Chronic Disease. Healthcare Policy. 2020; 5: 139-156.

15. Lewis A, Harding K, Snowdon D, Taylor N. Reducing wait time from referral to first visit for community outpatient services may contribute to better health outcomes: a systematic review. BMC Health Services Research. 2018; 18.

16. Bartolo M, Intiso D, Lentino C, Sandrini G, Paolucci S, Zampolini M. Urgent Measures for the Containment of the Coronavirus (Covid-19) Epidemic in the Neurorehabilitation/Rehabilitation Departments in the Phase of Maximum Expansion of the Epidemic. Frontiers in Neurology. 2020; $11: 1$.

17. Quick guide: Discharge to assess. The Department of Health. 2020.

18. McNeary L, Maltser S, Verduzco-Gutierrez M. Navigating Coronavirus Disease 2019 (Covid-19) in Physiatry: A CAN Report for Inpatient Rehabilitation Facilities. PM\&R. 2020; 12: 512-515.

19. Haines K, Berney S. Physiotherapists during COVID-19: usual business, in unusual times. Journal of Physiotherapy. 2020; 66: 67-69.

20. Prvu Bettger J, Thuomi A, Marquevich V, De Groote W, Rizzo Battistella L, Inamura M, et al. COVID-19: maintaining essential rehabilitation services across the care continuum. BMJ Global Health. 2020; 5: 2670.

21. Jourdan C, Azouvi P, Vittala E, Timlin S, Tenovuo O. Self-perceived care 
needs and barriers to care for persons with chronic traumatic brain injury. Annals of Physical and Rehabilitation Medicine. 2017; 60: 46.

22. Shannon M, Lispon-Smith R, Elf M, Olver J, Kramer S, Bernhardt J. Bringing the single versus multi-patient room debate to vulnerable patient populations: a systematic review of the impact of room types on hospitalized older people and people with neurological disorders. Intelligent Buildings International. 2018; 1: 1-19.
23. Mazza A, Paneroni M. Gym space in the era of digital cardiovascular rehabilitation: Often overlooked but critically important. European Journal of Preventive Cardiology. 2019; 27: 2059-2062.

24. Vishwanath A, Monga P. Rapid review and recommendations for MSK and allied heath personnel. Journal of Clinical Orthopedics and Trauma. 2020; 11: $500-503$ 